\title{
The Impact of Covid 19 Quarantine on the Intellectually Disabled
}

\author{
Rhoda L Ross-Williams \\ Independent Author, Advocate and Researcher, USA \\ Correspondence should be addressed to Rhoda LRoss-Williams, rosswilliamsrhoda@gmail.com
}

Received: July 25, 2020; Accepted: August 10, 2020; Published: August 18, 2020

\begin{abstract}
The COVID-19 quarantine is creating a threat to the intellectually disabled community. There is a need for community outings to sustain the psychological wellbeing of the intellectually disabled. This is why their caregivers include community outings in their care. However, during the COVID-19 quarantine this is suddenly missing in their lives. Therefore, this is causing an increase in psychosis for the intellectually disabled. Mental health professionals must proceed with caution when providing medication to ensure the holistic wellbeing of the patients is met.
\end{abstract}

\section{KEYWORDS}

Mental health; COVID-19; Intellectually disabled

\section{INTRODUCTION}

Holistic health requires medical professionals to consider the psychological wellbeing of the public during a crisis. People become anxious when they are under quarantine. Therefore, the intellectually disabled (ID) become more anxious [1]. They require psychological and medical needs to foster their best interests. The lives of many people did change as the COVID-19 pandemic became a major health threat requiring quarantine in America. This means many schools and community programs did shut down. This is a very abrupt change for the intellectually disabled especially for such an extensive amount of time. Therefore, many intellectually disabled people are having psychological issues from the quarantine because they need socialization to thrive.

\section{INTELLECTUAL DISABILITY}

The disabled community consists of many types of disability. This article will focus on the intellectually disabled spectrum. Anyone with an IQ below 70 has an intellectual disability along with their primary disability [1]. Therefore, they have difficulty with daily living, communication, socialization, and self-care. This is why they need community programs and public education to teach them adaptive behavior and appropriate behaviors. Many ID patients under the age of 21 are in special education program in their public school system. They have an individualized education plan (IEP). This is a part of the individuals with disabilities education act (IDEA). 
This act provides the support disabled student $\mathrm{s}$ need to thrive at school and in the community. This is why the quarantine is difficult for ID patients. The programs for ID patients have a lot of socialization and community based learning [2]. Therefore, the new normal is not a good health practice for the intellectually disabled. They function on routine and habit meaning they do not adapt well to sudden change [1]. The COVID-19 quarantine is a sudden change that seems to have no ending. This is extremely hard for children with intellectually disabilities because they have no socialization or community based programs. This can lead to psychosis and many bad behaviors.

\section{PSYCHOSIS FROM QUARANTINE}

Individuals with ID are deficient in their socialization, empathy and other normal psychiatric behaviors. Therefore, their lack in cognitive abilities causes them to have a difficult time accepting sudden changes in their daily routines [2]. This causes psychosis, severe mental disorders occurring with no connection to reality [2]. Therefore, ID patients begin to have emotional breakdowns and rage when they feel someone is keeping them away from their daily routine. This rage can lead to violence against others especially the caregivers.

Caregivers' roles in ID patients' daily lives is to keep them on a daily schedule to maintain their well-being. Many ID patients daily care plan consists of a consistent schedule and caregivers teach them to learn their schedule [2]. This means they are used to doing things one way and a change requires an understandable explanation. Therefore, a sudden change in schedule with no parameters of when the schedule will return to normal does trigger many psychological disorders causing psychosis for ID patients [1]. This is why quarantine with no ending is very unhealthy for children and adults with IDs.
They have many frustrations from being in a quarantine that took away their schooling and community programs. Their mental capacity makes it difficult for them to comprehend the never ending confinement to their residence [3]. Holistic health and wellbeing is difficult for caregivers during the COVID-19 pandemic because many IDs patients are more subject to catch the pathogen because they do not understand distance, keeping their hands out their face, and not touching things.

Their frustration leads to aggressive and destructive behaviors in their residence. This forces many caregivers to seek psychotropic medication to contain the IDs patients psychosis which is very difficult as well during COVID-19 restrictions [3]. Psychiatrists are utilizing telecommunications to conduct appointments with patients before writing prescriptions to contain their behavior [3]. Psychiatrists are practicing conventional medicine as much as possible during the COVID-19 quarantine. This is a challenge for all parties patient, caregiver, and the psychiatrist.

This is a challenge for prescribing doctors because assessing the patient during a telecommunication session is difficult. IDs patients do not have good socialization skills so telecommunications does leave the psychiatrist codependent on the caregivers' description of the patients' behaviors [3]. Therefore, psychiatrists must also go into the psychological issues IDs patients are experiencing during the COVID-19 quarantine knowing that IDs patients do not like drastic sudden changes in their routines [3]. This means that their community activities for socialization are taken away causing their psychological breakdowns. Therefore, the psychiatrists have to practice ethically and avoid over prescribing medications.

It is not a good practice for psychiatrists to prescribe psychotropic medications without a full assessment of the patients' antecedents and triggers. This means 
professionally that they know the behaviors are the result of the COVID-19 quarantine and must prescribe or not prescribe medication with caution [3]. This means chemical restraints can easily become abusive to IDs patients during the COVID-19 quarantine. In addition, not prescribing a medication to calm the patient is dangerous for their wellbeing as well leaving them vulnerable for self-harm or abuse by caregivers.

The wellbeing of the IDs patients during the COVID-19 quarantine are at risks as they are self-harming or victims of abuse by caregivers. However, the wellbeing of caregivers is also a concern as IDs patients are very aggressive during the psychosis they experience during the quarantine [1]. This raises the concern that the four- month quarantine for COVID-19 with a 99\% survival rate is very dangerous for way more IDs patients and their caregiver than a pathogen with a $99 \%$ recovery rate.

\section{CONCLUSION}

COVID-19 is a pathogen that requires precaution. However, the survival rate of $99 \%$ versus the increase in abuse rates for the IDs community and their caregivers is greater than one percent [3]. Therefore, protecting people from the dangers of COVID-19 requires medical professionals to seek input from psychiatrists as well to understand the threats a long-term quarantine creates for the vulnerable IDs community. In conclusion, all medical practices during COVID-19 should holistically meet the needs of everyone especially the IDs community.

\section{REFERENCES}

1. Sappok T, Diefenbacher A, Winterholler M (2019) The Medical Care of People With Intellectual Disability. Deutsches Aerzteblatt International 116(48): 809-816.

2. Siegel M, McGuire K, Veenstra-VanderWeele J, et al. (2020) Practice parameter for the assessment and treatment of psychiatric disorders in children and adolescents with intellectual disability (Intellectual Developmental Disorder). Journal of the American Academy of Child \& Adolescent Psychiatry 59(4): 468-496.

3. Herat M (2020) "I feel like death on legs": COVID-19 isolation and mental health. Social Sciences \& Humanities Open 2(1): 100042 . 